\title{
NEW TRENDS OF BIOMETRIC SYSTEMS BASED ON FINGERPRINT
}

\section{Edin Ćatović, Saša Adamović}

University of Sinergija, Bijeljina, The Republic of Srpska, B\&H
Correspondence:

Edin Ćatović

e-mail:

cattovic@gmail.com

\begin{abstract}
:
Generally speaking, biometrics can be defined as a recognition of individuals based on their behavior or biological unique characteristics. Fingerprints are taken by pressing a finger against a paper or platen surface on a sensor. However, due to skin deformations, external influences, irregularities in the sensor area, etc., we often have incomplete or degraded sample images. When this happens, many issues within institutions that use this kind of technology can occur. For example, employees cannot check-in or checkout, which can sometimes influence important deadlines. As a result of many years of research, a whole new generation of Touchless 3D scanning devices was developed and is already available on the market. This new technology of scanning does not have these issues and it functions in a similar way, although it has many significant improvements. In this paper, a brief comparison of original methods, two-dimensional and three-dimensional biometric systems will be presented. [1] Hardware and software solutions will also be analyzed. Some of the key problems, such as shadows, background, reflections, sample irregularities, etc. will be mentioned as well.
\end{abstract}

Key words:

biometrics, fingerprint scanning, touchless, $3 \mathrm{~d}$ fingerprint, recognition systems.

\section{BIOMETRICS}

Biometrics is a science that deals with the measurable physical characteristics and/or behavioral characteristics. This term comes from the Greek words bios (life) and metron (measure). Standardized definitions of biometrics are "automated identifying individuals based on their behavioral and biological characteristics" (Bidgoli, 2006, p. 498). Some of the most common biometric physical characteristics include: fingerprints, hand geometry, iris, retina, facial features, thermograf face, vascular patterns (arrangement of veins), smell and DNA. The examples of behavioral biometrics include: signing, contracts, walking and typing on the keyboard. Biometrics were regulated and defined in accordance with the ISO (International Organization for Standardization) requirements. [2]

Biometrics relies on the recognition of keystroke, gait, voice, signature, and unique biological characteristics like face, retina, iris, hand geometry, DNA, palmprint, etc. Fingerprint biometrics has a wide range of applications in the areas of civil society, military industry, legal proceedings, etc. One of the most important methods that biometrics realies heavily on is the Fingerprint Scanning. [3] This biometric method is based on 
enrollment databases, which are used in protection of our country borders, criminal identitications and identity recognition. Figure 1. describes and compares different types of biometric characteristics.

\begin{tabular}{lllll}
\hline Type & Stability & Accuracy & Signal acquisition & Applications \\
\hline $\begin{array}{l}\text { Physiological } \\
\text { characteristics }\end{array}$ & $\begin{array}{l}\text { Relatively } \\
\text { stable }\end{array}$ & Accurate & Easy & $\begin{array}{l}\text { Widely applicable to all } \\
\text { three functions of } \\
\text { biometrics }\end{array}$ \\
$\begin{array}{lllll}\text { Behavior } \\
\text { characteristics }\end{array}$ & Not very & Somewhat & Easy & $\begin{array}{l}\text { Mostly used for } \\
\text { authentication, or combined } \\
\text { sith other biometrics for }\end{array}$ \\
& accurate & & authentication/identification \\
$\begin{array}{l}\text { Psychological } \\
\text { characteristics }\end{array}$ & Very & Not & Very difficult & $\begin{array}{l}\text { It is still in the early stage of } \\
\text { development. Possibly used } \\
\end{array}$ \\
& accurate & & $\begin{array}{l}\text { for spoof protection in } \\
\text { extreme high security } \\
\text { environment }\end{array}$ \\
\hline
\end{tabular}

Figure 1. Biometric characteristics types comparasion.

\section{BIOMETRIC SYSTEMS}

The traditional security systems are based on swipe/ ID cards, passwords, keys, tokens, and identity cards that proove a person's identity. As a result, passwords are often forgotten, stolen or used by an unauthorized person to access various private data, bank accounts, cars, houses, etc. Due to the rising importance of the IT in people's lives, new ways of data and bank account protection, that do not require memorizing numerous passwords, are on a high demand. One of the recently developed technologies for this purpose is biometrics. [4]

Biometric systems are mostly based on two steps, biometric enrollment (Figure 2.) and biometric recognition (Figure 3.)

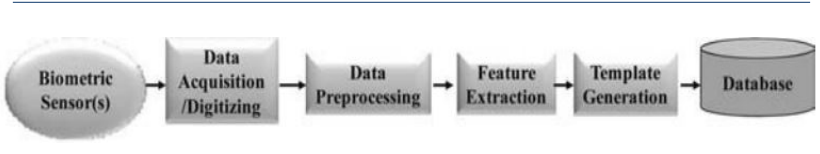

Figure 2. Biometric enrollment process

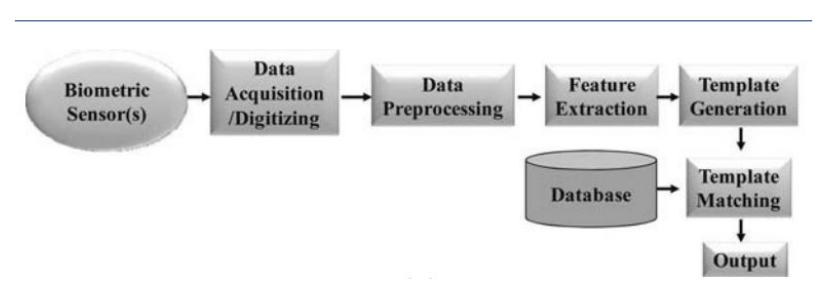

Figure 3. Biometric matching process.

Biometric technologies require a certain level of cooperation from users that is, i.e. users need to standstill while the devices scan their irises, faces, hands or fingers.
Iris systems are typically used for distances shorter than $30 \mathrm{~cm}$ and in front of cameras. Therefore, during the process of recognition, users must be motionless and look direcly at the camera. As these devices are based on infrared illumination techniques, this type of recognition does not pose any health risks.

On the other hand, recognition systems based on fingerprints and hand characteristics require from users to touch sensor surface and make contact in order to take a sample. This contact is sometimes characterized as unhygienic and some people do not want to use it. This and the fact that the fingertip sample can be misused, calls into question social acceptability of this method. pagination anywhere in the paper. Do not number text heads-the template will do that for you. [5]

Besides the above-mentioned reqirements, the presence of dirt on the contact surface can sometimes cause issues while taking fingerpint samples. Also, dirt can reduce the accuracy of the recognition process. Different finger pressures on the sensor can also make nonlinear distortions on the taken sample.

\section{NEW TRENDS OF BIOMETRIC SYSTEMS}

Touchless fingerprint recognition is a set of highaccuracy recognition techniques that help reduce issues while taking biometric samples. This also increases usability of devices and reduces the hardware costs. These systems can be applied in many areas, from acess control to high security required in goverments, military, research centers, etc.

One of the goals of this analysis is to present the areas in which these technologies can be used in the coming period. Our working hypothesis is that, although there are many others, the most important and lucrative field in which these technogies should be used is tablet and phone security systems, as well as ever more important the Internet of things.

In order to make biometrics more applicable and acceptable, researchers are developing new techniques. For example, iris recognition systems that can be used from a greater distance and while users are in motion, as well as touchless biometric systems for fingerprint and hand.[6]

The anaylsed touchless biometric technologies are based on few cameras and two-dimensional and threediomensional fingerprint samples. Compared with the taditional biometric fingerprint use, this new technology provides more security, scalability, accuracy and is socially more acceptable. 
It is important to mention that we have two techniques of touchless fingerprint based systems. First, twodimensional systems, and second, three-dimensional systems. The difference between these systems is the classification of samples that are taken. The first method uses a single CCD camera for taking sample images, while the three-dimensional system uses more complex hardware to collect sample images and, as a result, it has a much better accuracy.

The majority of biometric applications and techniques are based on controlled procedures. For example, users have to touch a sensor or be motionless over the course of recognition or sampling. The developer's goal is to provide users with the most comfortable technology.

Less-Constrained Biometric Systems aim to:

- increase the distance between the users and sensors

- reduce the required level of user co-operation

- design a recognition method compatible with uncontrolled light conditions

- design highly usable adaptive acquisition systems

- design pre-processing methods for reducing noise and enhancing data captured under less constrained conditions

- develop new feature extraction and matching algorithms specifically designed to obtain accurate results, using data captured under less constrained scenarios

- design method that permits data compatible with the existing biometric technologies to be obtained from samples captured under less constrained applications.

\section{TOUCHLESS FINGERPRINT BIOMETRICS, FEATURES AND TYPES}

\section{Touchless Biometric Features}

Biometric features traditionally captured using touchless sensors are suitable for use in non-restricted recognition systems. Many studies have examined the reduction of limitations in these systems. Two of the most researched technologies consist of less restricted biometric systems based on the iris and face scanning.

Face recognition systems cannot provide sufficient level of security. The iris is considered to be the most accurate biometric feature and can be used with the assistance of CCD cameras. [1]

\section{Touchless vs traditional fingerprints}

Fingerprint biometrics is one of the most common biometric features in biometric techniques, due to its favorable characteristics: durability, user-friendliness and distinctiveness. The analysis of fingerprint samples can be performed on 3 levels. The first level is so-called global, the second level is called thin and the third level is ultra. These analyses usually include acquisition, quality evaluation, enhancement, feature extraction, and matching. Difference between traditional and touchless fingerprint is shown in Figure 4.

Most of fingerprint recognition systems use sensors that require contact with their surface. However, these systems often have issues specifically due to this contact (i.e. distortions in the captured images and latent fingerprints on the acquisition surface).[1] In order to overcome these problems and increase social acceptability of the biometric recognition processes, touchless recognition systems are being extensively analyzed. These systems are based on CCD cameras and can be classified into systems based on two-dimensional and three-dimensional fingerprint samples. Systems like these, in most cases, are supposed to have support for existing API-s.[7]
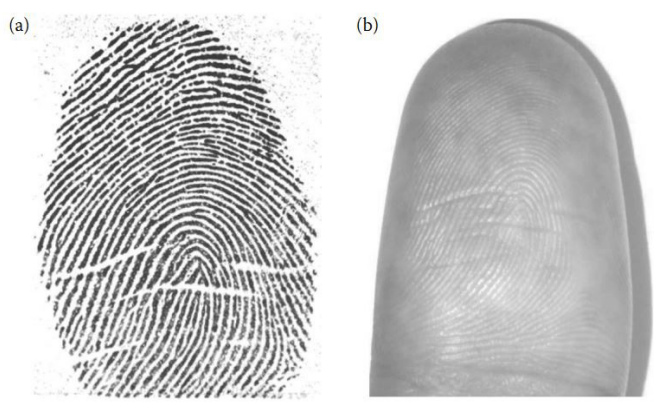

Figure 4. Traditional (a) vs. touchless fingerprint (b).

\section{Touchless Fingerprint Biometrics}

This system has three levels of accuracy check. Complete application of this type of fingerprint is illustrated in Figure 5.

- First level: The overall global ridge flow pattern is recognized.

- Second level: The recognition is based on distinctive points of the ridges, which are

- called minutiae points.

- Third level: Ultrathin details, such as pores and incipient ridges, are recognized. 


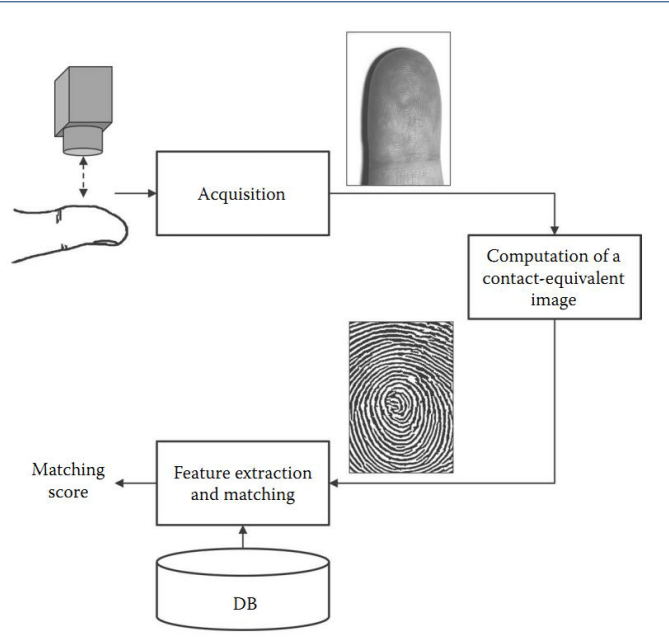

Figure 5. Application of touchless fingerprint system.

\section{Touchless two-dimensional fingerprints}

The analysed touchless fingerprint recognition systems include those based on two-dimensional systems designed to be integrated in low-cost and portable devices and those using more complex hardware setups in order to obtain higher-recognition accuracy.

Touchless fingerprint recognition systems based on two-dimensional samples use CCD cameras to capture details of the finger ridge pattern. These systems usually capture a single image and then process it in order to obtain a contact-equivalent fingerprint image. The purpose of this process is to compute a fingerprint image and make it compatible with the existing biometric recognition systems designed for touch-based images. Then, a biometric recognition is performed using well known algorithms for extracting features and matching touchbased fingerprint images. Other systems use features extraction and matching algorithms specifically designed for touchless two-dimensional samples. Some systems also use multiple-view techniques or specifically designed optics in order to obtain touch-equivalent fingerprint images not affected by perspective deformations and focusing problems. Some samples of two-dimensional fingerprints are shown in Figure 6.

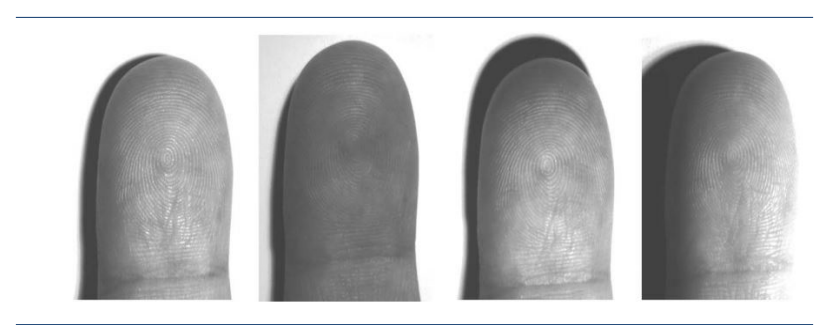

Figure 6. Two dimensional touchless fingerprint samples.

\section{Touchless three dimensional fingerprints}

Compared with biometric systems based on twodimensional samples, systems that compute three-dimensional fingerprint models use more information and less distorted data. In fact, the analysed samples consist of three-dimensional structures that are not affected by perspective deformations and that show only the fingertip (Figure 7). Moreover, the feature extraction and matching algorithms can use additional information related to with the $z$-axis to improve the recognition accuracy. However, these systems require acquisition setups that are more complex and expensive than those for single Touchless images. Moreover, most reported methods require complex acquisition procedures. Figure 8. illustrates the scheme of three-dimensional fingerprint [8].

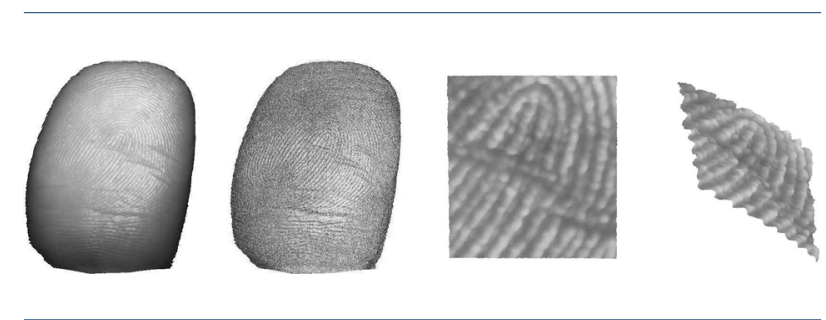

Figure 7. Example of $3 \mathrm{~d}$ fingerprints (texture, depth rendering).

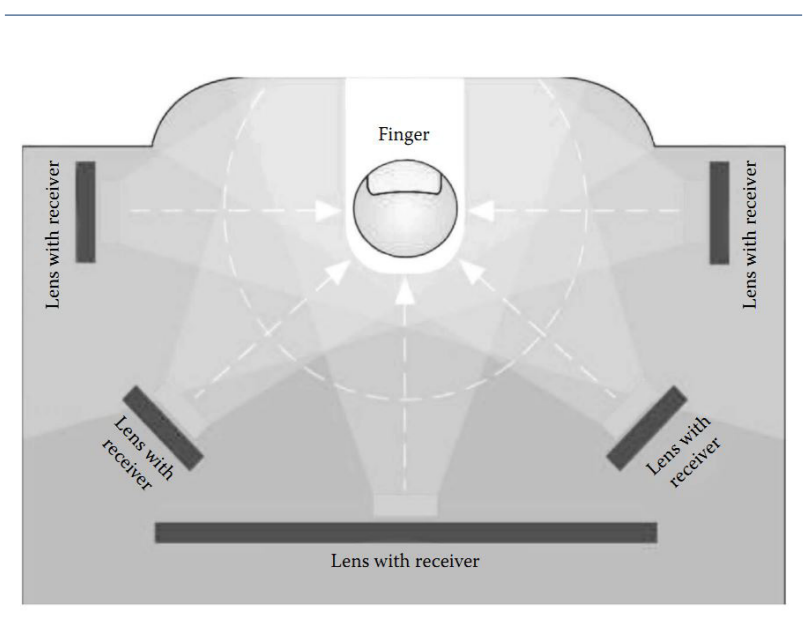

Figure 8. Three-dimensional scheme.

\section{CONCLUSION}

This paper presents new multidisciplinary technologies for Touchless fingerprint recognition compatible with less-constrained scenarios compared with traditional biometric systems. It is necessary to implement multidisciplinary approach to understand and implement this. The 
described technologies include all aspects of Touchless biometric recognition systems based on two-dimensional and three-dimensional fingerprint samples, including methods for acquiring and processing biometric data.

New technologies were described and compared to the traditional ones. Also, they are comparable in many as pets as accuracy, speed, cost, scalability, interoperability, usability, social acceptance, security, and privacy.

The results that will tell us that in high-security application, Touchless biometric systems based on threedimensional samples could achieve better accuracy than traditional touch-based fingerprint recognition systems.

On the other hand, two-dimensional solutions can be applied in some low cost systems, such as mobile devices. Therefore, the research demonstrated that touchless fingerprint recognition methods can be used effectively in different application scenarios where we have live touch-based techniques implemented.

\section{REFERENCES}

[1] Ruggero Donida Labati, Vincenzo Piuri, and Fabio Scotti (2015), Touchless Fingerprint Biometrics, Taylor \& Francis Group, LLC;

[2] Phillip Wiggin, Lars Ericson, Ph.D. (2014), Contactless Fingerprint Technologies Assessment (Version 2);

[3] Yi Chen,Geppy Parziale, Eva Diaz-Santana and Anil K. Jain, Compatibility with Legacy Rolled Images, 2006;

[4] "Mitsubishi Touchless Fingerprint Sensor," "http:// global.mitsubishielectric.com";

[5] Parziale G. and Diaz-Santana E, January 2006 ., "The Surround Imager: a Multi-Camera Touchless Device to Acquire 3D Rolled-Equivalent Fingerprints," in Proceedings of IAPR International Conference on Biometrics (ICB); Hong Kong, China;

[6] Tsai, R., 1986, "An Efficient and Accurate Camera Calibration Technique for 3D Machine Vision," in Proceedings of IEEE Conference on computer Vision and Pattern Recognition, Florida, USA;

[7] Edited by ELIZA YINGZI DU, 2012, Biometrics from fiction to practice, CRC Press Taylor \& Francis Group;

[8] "http://www.vis.uky.edu/ realtime3d/Research/ Fingerprint.htm"; 